\title{
UNIFORM DISTRIBUTION ON LOCALLY COMPACT GROUPS
}

\author{
HARALD RINDLER
}

\begin{abstract}
In this paper we give a short proof of the existence of uniformly distributed sequences in separable locally compact groups, a result first stated by Benzinger [1]. The existence of u.d. sequences in separable compact groups was proved by Veech [7] whose results also imply the above result. We also prove the existence of well-distributed sequences.
\end{abstract}

H. Weyl's classical notion of uniform distribution admits an extension to arbitrary locally compact groups [6, Chapter $4, \S 5]$.

The main result of this paper is the following Theorem (compare also [7, Definition, p. 125] and [1, "key lemma", p. 150]).

THEOREM. Let $G$ be a locally compact group, $C$ a class of nontrivial, continuous, unitary, irreducible, finite-dimensional representations of $G$. If there exists a countable subset $F$ of $G$ such that $U(F)$ is dense in $U(G)$ for each $U \in C$ (in the usual topology), then there exists a sequence $\left(x_{n}\right)$ such that: For each $U \in C$ and any $\varepsilon>0$, there exists $M=M(U, \varepsilon)$ such that

$$
\left\|\frac{1}{N} \sum_{n=k+1}^{n=k+N} U\left(x_{n}\right)\right\|<\varepsilon \text { for all } N \geqslant M, \quad k=0,1,2, \ldots
$$

$(\|\cdot\|=$ Hilbert-Schmidt norm).

Proof. Let $B_{1}, B_{2}, \ldots$ be an enumeration of all finite subsets of elements of $F$. If $B=\left(x_{1}, \ldots, x_{m}\right)$ put

$$
(B, U)=\frac{1}{m}\left\|\sum_{n=1}^{m} U\left(x_{n}\right)\right\| .
$$

Using the fact that $U(x)=\left(u_{i, j}(x)\right)$ where the $u_{i, j}$ may be considered as continuous functions on the Bohr compactification of $G$ such that $\int u_{i, j}(x) d x$ $=0$ (" $d x$ " denotes Haar measure), or using [3, Theorem 3.8.4], we obtain

$$
\inf _{n}\left(B_{n}, U\right)=0 \text { for each } U \in C .
$$

If $B=\left(x_{1}, \ldots, x_{m}\right), D=\left(y_{1}, \ldots, y_{k}\right)$ put

Received by the editors July 1, 1974 and, in revised form, April 28, 1975.

AMS (MOS) subject classifications (1970). Primary 22D05; Secondary 10K99.

Key words and phrases. Locally compact groups, uniform distribution, finite-dimensional unitary representations, Bohr compactification. 


$$
B D=\left(x_{1} y_{1}, x_{1} y_{2}, \ldots, x_{1} y_{k}, x_{2} y_{1}, \ldots, x_{m} y_{k}\right) .
$$

Considering the relations $(a B, U)=(B a, U)=(B, U)$ (where $a B$ $\left.=\left(a x_{1}, \ldots, a x_{m}\right), a \in G\right),\|A V\|=\|V A\|=\|A\|$ for unitary $V$ and arbitrary $A$, and $B D=\left(x_{1} D, x_{2} D, \ldots, x_{m} D\right)$, and also using the fact that $B D$ represents the same set as $\left(B y_{1}, B y_{2}, \ldots, B y_{k}\right)$, we obtain

$$
(B D, U) \leqslant(B, U),
$$

$(B D, U) \leqslant(D, U)$ for all $U$.

Replacing the block $B_{n}$ by a block $z_{n} B_{n}$ we may assume that $B_{n}=(1, \ldots)$. If we define $A_{m}$ successively by $A_{1}=B_{1}, A_{m+1}=B_{m+1} A_{m}$ then we have $A_{1} \subseteq A_{2} \subseteq \cdots$, and obtain a sequence $\left(x_{n}\right)$ such that the block consisting of the first $K_{m}$ (= number of elements of $A_{m}$ ) terms coincides with $A_{m}$.

Given $\varepsilon>0, U \in C$, then by (1) and (2) for a suitable $m$ we have $\left(A_{m}, U\right)<\varepsilon / 2$. We have $\left(x_{1}, x_{2}, \ldots, x_{n}, \ldots\right)=\left(A_{m}, z_{2} A_{m}, z_{3} A_{m}, \ldots\right.$, $\left.z_{j} A_{m}, \ldots\right)$ for suitable $z_{j}$ 's. Therefore any sequence $\left(x_{k+1}, \ldots, x_{k+N}\right)$ can be divided into " $A_{m}$-like" blocks with the exception of at the most $K_{m}$ terms. If $U$ has dimension $r(\|U(x)\|=\sqrt{r}$ for all $x$ in $G)$ then we obtain for $N \geqslant 2 K_{m} r^{1 / 2} / \varepsilon=M(U, \varepsilon)$,

$$
\left\|\sum_{n=k+1}^{k+N} U\left(x_{n}\right)\right\| \leqslant K_{m} \sqrt{r}+\left(N / K_{m}\right) K_{m}\left(A_{m}, U\right)<N \varepsilon .
$$

\section{Q.E.D.}

CoNSEQuences. If $G$ is a locally compact group then a sequence $\left(x_{n}\right)$ is called uniformly distributed in $G$ if for every closed normal subgroup such that $G / H$ is compact, the sequence $\left(x_{n} H\right)$ is u.d. in $G / H$ (Rubel [6, Chapter 4 , Definition 5.2]). $G$ is called $K$-separable if there exists a countable subset $F$ such that $F H$ is dense in $G / H$ for any $H$ as above. The following result of Benzinger [1, Theorem 3.6] is an immediate consequence of our Theorem and Weyl's criterion [6, Chapter 4, Theorem 5.5].

Corollary 1. A locally compact group possesses a u.d. sequence if and only if $G$ is $K$-separable.

Corollary 1 is also a consequence of a deep result of Veech [7, Theorem 3]. He proves the existence of a sequence of positive integers $\left(r_{n}\right)$ (uniformly distributed sequence generator) such that for any compact group $K$ and any sequence $\left(y_{n}\right)$ in $K$ which is contained in no proper closed subgroup, the sequence $x_{n}=y_{r_{1}} y_{r_{2}} \cdots y_{r_{n}}$ is u.d. in $K$. If $G$ is $K$-separable there exists a sequence $\left(y_{n}\right)$ such that $\left(y_{n} H\right)$ is dense in $G / H$, whenever $G / H$ is compact; and the generated sequence $\left(x_{n}\right)$ is u.d. in $G$.

If $G$ is compact then $\left(x_{n}\right)$ is called well distributed if

$$
\lim \frac{1}{N} \sum_{n=1}^{N} f\left(x_{n+k}\right)=\int f(x) d x
$$

for any continuous $f$, uniformly in $k$ (see [6, Chapter 4, Definition 1.1, Lemma 1.1]). If $N$ denotes the intersection of the kernels of all continuous, unitary, finite-dimensional representations of $G$ ("von Neumann-kernel"), and if $b: G \rightarrow b G$ denotes the Bohr homomorphism from $G$ into the Bohr compacti- 
fication, then we obtain by our Theorem and by Weyl's criterion for welldistributivity [6, Chapter 4, Corollary 1.3]

COROllary 2. If $G / N$ is separable then there exists a sequence $\left(x_{n}\right)$ in $G$ such that $b\left(x_{n}\right)$ is well distributed in $b(G)$.

Both corollaries (for u.d. sequences) were proved in [2] for Abelian groups. A sequence $x_{n}$ in $G$ such that $b\left(x_{n}\right)$ is uniformly distributed in $b(G)$ is called Hartman u.d. We note that the construction of a Hartman u.d. sequence given in [2] actually yields a well-distributed sequence although the concept of welldistribution is not considered in that paper.

We mention the question of when the existence of a Hartman u.d. sequence implies the separability of $G / N$. For Abelian groups where, of course, $G / N=G$, this implication follows from Proposition 2.4 and Theorem A (Kakutani) of [2]. If $G$ is almost connected, that is $G / G_{e}$ is compact where $G_{e}$ is the connected component of the identity, then we see that the existence of a Hartman u.d. sequence implies the separability of $G / N$ by using the fact that $G / N$ contains an open subgroup (of finite index) isomorphic to $R^{n} \times K, K$ compact (see [4, Theorem 2.9(2)] and [5, VII, \$2, 7.2.1]). We do not know, in general, whether there exists $G$ such that $G / N$ is nonseparable and yet $G$ admits a Hartman u.d. sequence.

We note in closing that if $G$ is Abelian and separable and if the set $C$ in our Theorem is, in addition, compact (as a subset of $\hat{G}$ ), then the index $M(U, \varepsilon)$ may be chosen independent of $U \in C$. A similar statement holds in the nonAbelian case.

\section{REFERENCES}

1. L. Benzinger, Uniformly distributed sequences in locally compact groups. I, Trans. Amer. Math. Soc. 188 (1974), 149-165. MR 48 \#11388.

2. I. D. Berg, M. Rajagopalan and L. A. Rubel, Uniform distribution in locally compact Abelian groups, Trans. Amer. Math. Soc. 133 (1968), 435-446. MR 37 \#3279.

3. F. P. Greenleaf, Invariant means on topological groups and their applications, Van Nostrand Math. Studies, no. 16, Van Nostrand Reinhold, New York, 1969. MR 40 \#476.

4. S. Grosser and M. Moskowitz, Compactness conditions in topological groups, J. Reine Angew. Math. 246 (1971), 1-40. MR 44 \#1766.

5. H. Heyer, Dualität lokalkompakter Gruppen, Lecture Notes in Math., vol. 150, SpringerVerlag, Berlin and New York, 1970. MR 43 \#11.

6. L. Kuipers and H. Niederreiter, Uniform distribution of sequences, Wiley, New York, 1974.

7. W. A. Veech, Some questions of uniform distribution, Ann. of Math. (2) 94 (1971), 125-138. MR 44 \# 4187.

Mathematisches Institut der Universität Wien, Strudlhofgasse 4, A-1090 Wien, AusTRIA 